\title{
Speeding up all-against-all protein comparisons while maintaining sensitivity by considering subsequence-level homology
}

Orthology inference and other sequence analyses across multiple genomes typically start by performing exhaustive pairwise sequence comparisons, a process referred to as "all-againstall". As this process scales quadratically in terms of the number of sequences analysed, this step can become a bottleneck, thus limiting the number of genomes that can be simultaneously analysed. Here, we explored ways of speeding-up the all-against-all step while maintaining its sensitivity. By exploiting the transitivity of homology and, crucially, ensuring that homology is defined in terms of consistent protein subsequences, our proof-ofconcept resulted in a $4 x$ speedup while recovering $>99.6 \%$ of all homologs identified by the full all-against-all procedure on empirical sequences sets. In comparison, state-of-the-art kmer approaches are orders of magnitude faster but only recover $3-14 \%$ of all homologous pairs. We also outline ideas to further improve the speed and recall of the new approach. An open source implementation is provided as part of the OMA standalone software at http://omabrowser.org/standalone. 


\title{
Speeding up all-against-all protein comparisons while maintaining sensitivity by considering subsequence-level homology
}

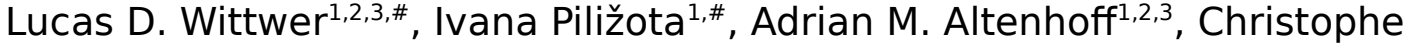 \\ Dessimoz ${ }^{1,2, *}$
}

${ }^{1}$ University College London, Gower St, London WC1E 6BT, United Kingdom ${ }^{2}$ Swiss Institute of Bioinformatics, Universitätstr. 6, 8092 Zurich, Switzerland ${ }^{3}$ ETH Zurich, Computer Science, Universitätstr. 6, 8092 Zurich, Switzerland

\#These authors contributed equally to this work.

*Corresponding author. Email: c.dessimoz@ucl.ac.uk

\begin{abstract}
Orthology inference and other sequence analyses across multiple genomes typically start by performing exhaustive pairwise sequence comparisons, a process referred to as "all-against-all". As this process scales quadratically in terms of the number of sequences analysed, this step can become a bottleneck, thus limiting the number of genomes that can be simultaneously analysed. Here, we explored ways of speeding-up the all-against-all step while maintaining its sensitivity. By exploiting the transitivity of homology and, crucially, ensuring that homology is defined in terms of consistent protein subsequences, our proof-of-concept resulted in a $4 x$ speedup while recovering $>99.6 \%$ of all homologs identified by the full all-against-all procedure on empirical sequences sets. In comparison, state-of-the-art $k$-mer approaches are orders of magnitude faster but only recover $3-14 \%$ of all homologous pairs. We also outline ideas to further improve the speed and recall of the new approach. An open source implementation is provided as part of the OMA standalone software at http://omabrowser.org/standalone.
\end{abstract}


33 Most approaches start by identifying genes of common ancestry within and across

34 species-sets of "homologous" genes (Patterson 1988). As further refinement,

35 homologous proteins, which can be split up into paralogs and orthologs, diverged

36 from a common ancestral protein (Fitch 1970). Paralogous sequences, which start

37 diverging through gene duplication, are believed to drive function innovation and

38 specialisation, whereas orthologous sequences, which diverged through speciation,

39 tend to have more similar biological function (Tatusov 1997; Lynch 2000; Altenhoff et

40 al. 2012; Gabaldón and Koonin 2013). Therefore, protein function can be assigned by

41 identifying regions of orthology between a newly sequenced and an already

42 annotated genome (e.g., Gaudet et al. 2011). Orthology inference is also of high

43 interest to infer phylogenetic species trees and to study the evolution of gene

44 families (Altenhoff and Dessimoz 2012).

45 Due to the high biological interest for orthology detection, many orthology inference

46 algorithms and associated databases have been developed (reviewed in Dessimoz

47 et al. 2012; Sonnhammer et al. 2014). Some of these can be large, with databases

48 comparing thousands of genomes of different species: eggNOG with currently 3686

49 genomes (Powell et al. 2013), Roundup with 2044 (DeLuca et al. 2012), OMA with

501613 genomes (Altenhoff et al. 2011) and OrthoDB with 1425 genomes (Waterhouse

51 et al. 2013).

52 To detect homology, these resources rely on all-against-all protein comparison. All

53 pairs of sequences are aligned using BLAST (Altschul et al. 1997) or dynamic

54 programming (Smith and Waterman 1981). They are then inferred as homologous if

55 their alignment score is above a certain threshold and alignment length constraints

56 are satisfied (Dessimoz et al. 2005; Roth et al. 2008). Even orthology databases

57 based on reconciled gene/species trees (Vilella et al. 2008) or hidden Markov model

58 profiles (Mi et al. 2013) typically start their procedure with an all-against-all step,

59 before proceeding to clustering.

60 However, the all-against-all procedure scales quadratically in the number of

61 sequences compared and hence rapidly becomes very costly. For instance, the all-

62 against-all phase in OMA, the resource developed in our lab, has already consumed

63 over 6.1 million CPU hours to date, despite the use of a highly optimised

64 implementation (Szalkowski et al. 2008) of the Smith-Waterman (1981) pairwise

65 alignment algorithm. Furthermore, in the all-against-all phase of OMA, about half of 
66 the time is spent on unrelated gene pairs (see Supplementary materials) which are

67 not used for further orthology inference.

68 Previous methods have been proposed to speedup the all-against-all phase, but they 69 tend to come at a cost of lower sensitivity. CD-HIT (Li and Godzik 2006), UCLUST 70 (Edgar 2010), and kClust (Hauser et al. 2013) are methods to reduce the complexity 71 by clustering the dataset based on the number of common or high-scoring words ( $k$ 72 mers) in each sequence. This works well to identify closely related sequences-e.g., 73 close paralogs or "redundant" sequences-but such approaches are less sensitive 74 than full dynamic programming alignment and thus tend to miss distant homologs.

75 Another approach was proposed in the Hieranoid method (Schreiber and

76 Sonnhammer 2013): by using the species tree as guide and iteratively merging 77 terminal sister taxa into ancestral genomes, the computational cost only grows 78 linearly with the number of genomes. However, reconstructing ancestral sequences 79 or profiles can prove challenging among distantly related species. Moreover, the 80 guide tree must be clearly defined and known, which is a problem for many 81 prokaryotes and eukaryotes.

82 Here, we present a new strategy for speeding up the all-against-all step, by first 83 clustering sequences and then performing an all-against-all within each cluster.

84 Crucially, in the clustering phase, the new approach accounts for residue-level 85 homology and not merely whole protein-level homology. As a result, sequences can 86 belong to multiple clusters. On sets of empirical bacterial and fungal protein 87 sequences, our new approach achieves a reduction in computational time of $\sim 75 \%$ 88 while maintaining the sensitivity of all-against-all procedure ( $>99.6 \%$ of all pairs 89 identified by all-against-all).

91 We start by motivating our method under the assumption of perfect input data, then 92 present the modifications required to perform well on real, imperfect data. We then 93 describe the $k$-mer methods we used as points of comparison. Finally, we present 94 the empirical datasets used to evaluate the new method.

$95 \quad 2.1 \quad$ Building homologous clusters from perfect data 
96 Typical downstream analysis of the all-against-all step requires an alignment score

97 for every significant pair of proteins (putative homologs). Therefore, aligning these

98 significant pairs seems difficult to avoid. In contrast, avoiding the alignment of unrelated pairs can potentially reduce the overall runtime of the all-against-all. One way to avoid some pairs is by using the transitive property of homology: typically101 we discuss pathological cases in the next section-if sequence $A$ is homologous to sequence $B$, and sequence $B$ is homologous to sequence $C$, then sequences $A$ and $C$ are homologous. A corollary of this is that if sequence $A$ is homologous to sequence $B$, and sequence $A$ is not homologous to sequence $C$, then sequences $B$ and $C$ are 105 not homologous.

106

107

108

109

110

111

112

113

\section{4}

115

116

117

118

119

120

121

122

123

124

125

126

127
Proof of corollary: Let S1 denote "A is homologous to B", S2 "B is homologous to $\mathrm{C}$ " and S3 "A is homologous to C". The transitivity of homology states that $\mathrm{S} 1 \wedge \mathrm{S} 2 \Rightarrow \mathrm{S} 3$. By material implication, that it is equivalent to $\urcorner(\mathrm{S} 1 \wedge \mathrm{S} 2) \mathrm{v}$ S3. De Morgan's law yields the equivalent expression ( $7 \mathrm{~S} 1 \vee 7 \mathrm{~S} 2$ ) v S3 which is, due to the associativity and commutativity of disjunction, equivalent to ( $7 \mathrm{~S} 1 \vee \mathrm{S} 3) \vee 7 \mathrm{~S} 2$. Using the De Morgan's law again, the expression transforms to the equivalent $\urcorner(\mathrm{S} 1 \wedge\urcorner \mathrm{S} 3$ ) $\vee\urcorner \mathrm{S} 2$. Finally, by material implication it is equivalent to the expression $\mathrm{S} 1 \wedge\urcorner \mathrm{S} 3 \Rightarrow \mathrm{7S} 2$.

Therefore, in this particular scenario, if we infer that $A$ and $B$ are homologous and $A$ and $C$ are non-homologous, there is no need to align sequences $B$ and $C$. Another way to consider this situation is that $A$ acts as a representative for its homologous sequence $B$. We can generalise this idea to more than three sequences by building clusters of homologs for which a single sequence acts as a representative for all members (trivial proof by induction).

Accordingly, we can split the all-against-all into two steps: (i) building homologous clusters, and (ii) performing the all-against-all within each cluster. In the clustering step, we initialise the procedure with a single cluster containing the first sequence as its representative and sequentially consider all the other sequences: each sequence is aligned to the representative sequence of each homologous cluster. If an alignment score is significant, implying that the sequence is homologous to members of that particular cluster, the sequence is added to the cluster. Else, a new cluster is formed with that sequence as representative. 
128 Under the assumptions that homology is transitive and that homology can be

129 perfectly ascertained through sequence alignment, this procedure guarantees that

130 all homologous sequences end up in a common cluster in $\mathrm{O}(\mathrm{nk})$, where $\mathrm{n}$ is the

131 number of proteins and $k$ is the number of clusters. Since $k \leq n$ (and possibly $k<<n$ ),

132 this is more efficient than the $\mathrm{O}\left(\mathrm{n}^{2}\right)$ runtime of the standard all-against-all procedure.

133 In practice, however, homology at the level of proteins is not always transitive and 134 homology cannot be perfectly ascertained. Therefore, refinements to the basic idea 135 are required.

136

137

138

139 140

141

142

143

144

145

146

147 Thus, to deal more effectively with real sequences, we modify the basic algorithm in 148 three ways. First, instead of a single representative sequence, we also consider a 149 variation of the algorithm that uses several representative sequences for each 150 cluster. This can be advantageous for homologous clusters containing very dissimilar 151 sequences, which are thus poorly represented by any single sequence. Second, we 152 allow for the possibility that some sequences might be homologous to more than 153 one cluster. This could be the case if homologous clusters are fragmented due to 154 high divergence among some of their members (and among their representatives in 155 particular). This also enables multi-domain proteins to be included in clusters 156 corresponding to different domains. Third, we take into account subsequence-level 157 homology: we ensure that the entire length of a particular protein (minus a tolerance 158 parameter, set to 20 amino-acid residues in this study) is covered by the cluster(s) 
159 to which the sequence belongs. If this is not the case, a new cluster is created with 160 the sequence as representative. Note that this is in addition to inclusion into any 161 cluster with significant alignment (Fig. 1B)).

A

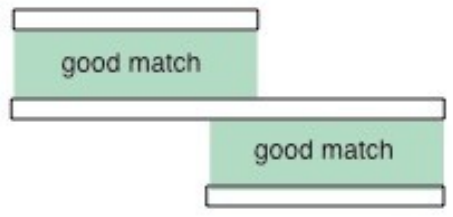

Transitivity of homology does not hold across inconsistent subsequences
B

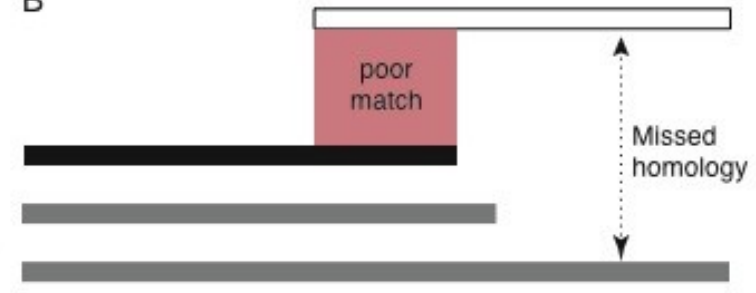

162

163

164

165

166

Figure 1: Diagram of potential problems with transitivity of homology. (A) The

transitivity of homology does not hold if the residues involved are inconsistent. (B) Homologous relationships can be missed if significant sequence parts are not covered by the representative sequence. To address this problem, our approach would create a new cluster, with the sequence that is not fully covered as its representative.

167 Putting these ideas together, our approach for building homologous clusters is as 168 follows. First, we sort the input genomes by their number of sequences in 169 descending order. For every genome, starting with the one with the highest number

170 of sequences, we process its sequences one by one as they are listed in the

171 database file. As there are no clusters at the beginning of the process, the first

172 sequence founds a cluster and becomes its representative. Every subsequent

173 sequence is aligned with the representatives of the current cluster(s) using Smith-

174 Waterman (1981). We run the vectorised Smith-Waterman implementation of

175 Szalkowski et al. (2008) using the 224 GCB scoring matrix (Gonnet et al. 1992). If

176 the score is above the minimum threshold of 135.75 , the sequence is added to the

177 cluster. Furthermore, if the number of cluster representatives in the particular

178 cluster is below the maximum allowed, the newly added sequence becomes a

179 representative. We also keep track how much the sequence is covered by the

180 representatives of the assigned clusters. We do not introduce any restrictions on the

181 size of the clusters nor on the number of clusters that a sequence is assigned to.

182 After exhaustive search through all cluster representatives, we assess whether the

183 sequence was added to one or multiple clusters. If the full length of the sequence 
184 (minus a tolerance of 20 amino acids) is not covered by the clusters to which the

185 sequence was added, an additional cluster is created with the sequence as a

186 representative. The same applies if the sequence was not assigned to any clusters

187 (see Box 1 for pseudocode).

188

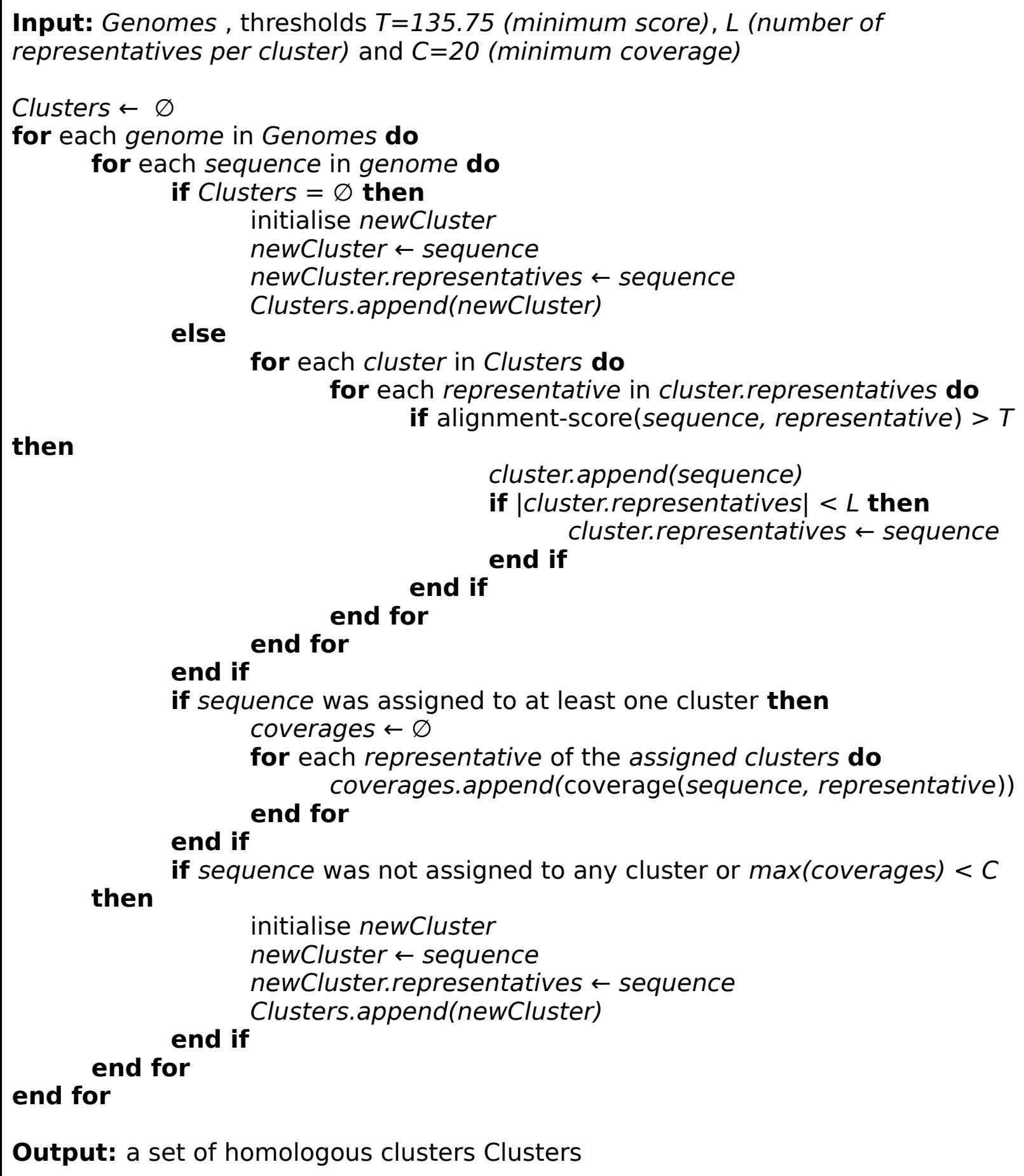

Output: a set of homologous clusters Clusters 
191 Once we have identified all homologous clusters, we compute an all-against-all

192 within each cluster using the same criteria as the global all-against-all (Figure 2).

193 This ensures that all retained pairs agree with the score and length requirements of 194 the full all-against-all, and provides scores for all homologous pairs, which are often 195 required for downstream analyses.

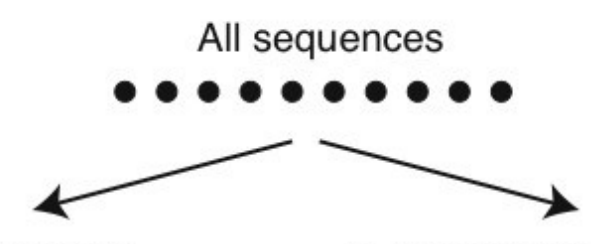

A. Existing approach

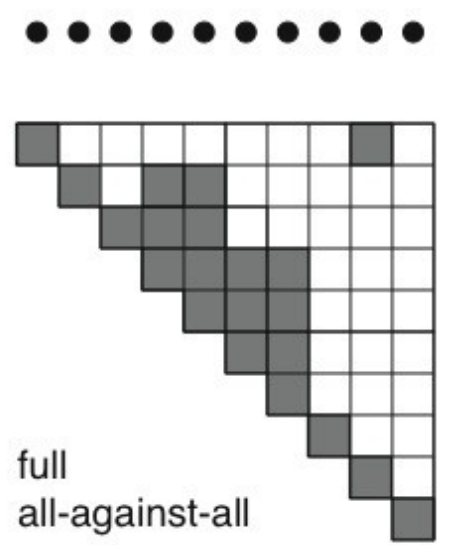

significant alignment
B. New approach with clustering
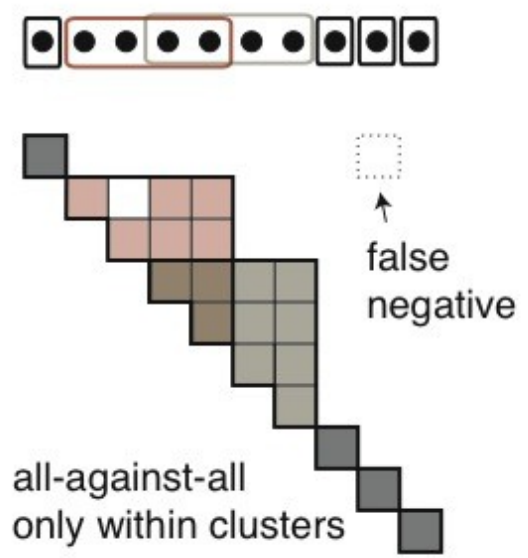

196

197

198

199

200

Figure 2. Comparison between the current and the new approach. Diagram of $(A)$ the current OMA all-against-all and (B) the new approach with homologous clustering and sequence coverage. In the proposed approach, the sequences are first clustered and then the all-against-all is run only within clusters. The overall number of computations is considerably reduced, but some homologous relationships can be missed.

202 The baseline of this study is given by the current all-against-all procedure in the 203 OMA database (Roth et al. 2008; Altenhoff et al. 2011). It runs a highly optimised 204 Smith-Waterman (1981) local alignment implementation (Szalkowski et al. 2008) 205 using the 224 GCB scoring matrix (Gonnet et al. 1992). To be inferred as homologs, 206 sequence pairs need to reach an alignment score of at least 135.75 with the 224 
207 GCB matrix (and 181 with an optimised GCB matrix, but this additional requirement 208 was not considered in this study), with the additional requirement that the length of 209 the shorter sequence aligned be at least $61 \%$ of the longer sequence. These

210 parameters were previously established to yield good results on empirical data (Roth 211 et al. 2008).

212 To compare the performance of the new approach to $k$-mer clustering, we also ran 213 kClust (Hauser et al. 2013) and UCLUST (Edgar 2010). We ran kClust with default set 214 of parameters, where the required minimum sequence identity was $30 \%(-s 1.12)$ 215 and the minimum alignment coverage of the longer sequence was $80 \%\left(\begin{array}{ll}-c & 0.8\end{array}\right)$. To 216 increase sensitivity, we also ran kClust with $30 \%$ as minimum for sequence identity $217(-s \quad 1.12)$ and $50 \%$ as minimum alignment coverage $(-c 0.5)$. The jobs were run on 218 a single core of a Dell R910 (Intel Xeon E7-8837, $2.66 \mathrm{GHz}$ ) with 32 cores and 1TB of 219 memory. For UCLUST, we used the recommended option -cluster_fast. We first 220 ran UCLUST with default parameters, specifying $30 \%$ as sequence identity (-id $2210.3)$. We also ran it with the additional requirements on coverage of the target 222 sequence being $50 \%$ (-target_cov 0.5 ) and allowing sequences to be assigned to 223 multiple clusters (-maxaccepts 0 ), without limiting the number of sequences 224 rejected before quitting search (-maxrejects 0 ). In both cases, only sequences 225 longer than 50 amino acid residues were taken into account-the same length 226 requirement as in the all-against-all procedure. UCLUST was run on a MacBook Air 227 (Intel Core i7, 1.7 GHz) dual-core with 8GB of memory.

\subsection{Datasets}

229 We analysed the performance of the new approach on two distinct datasets:

230 bacteria and fungi. Bacteria dataset contained 14 proteomes, fungi contained 12, all 231 sampled from the OMA database (Altenhoff et al. 2011) (March 2014 release; see 232 Supplementary File 1 for complete list). The distribution of sequence lengths and 233 evolutionary distances among significant pairs is provided in Supplementary

234 materials Fig. 1-2. To assess the scaling behaviour of each variant, we ran them on 235 subsets which were formed as follows: all genomes in the dataset were sorted by 236 their size and the ones in the middle of the list were chosen to form a subset. By 237 choosing the central 2, 4, 6, 8, 12 and finally all 14 proteomes, subsets of bacteria 238 were constructed. We repeated the procedure for fungi and obtained subsets of size $2392,4,6,8,10$ and 12 proteomes. 
240 In addition, we performed an extra analysis on a diverse dataset containing two

241 vertebrates, one plant, one protist, one fungus, and one bacterium (Supplementary

242 File 1).

\section{$243 \quad 3$ Results}

244 To assess the time and accuracy of our new approach, we compared the four

245 clustering variants-one or three cluster representatives, each combined with either

246 taking into account residue-level homology or not (see Methods) - on empirical

247 bacterial genomes. We also repeated the three fastest variants on empirical fungal

248 datasets.

A. Bacteria

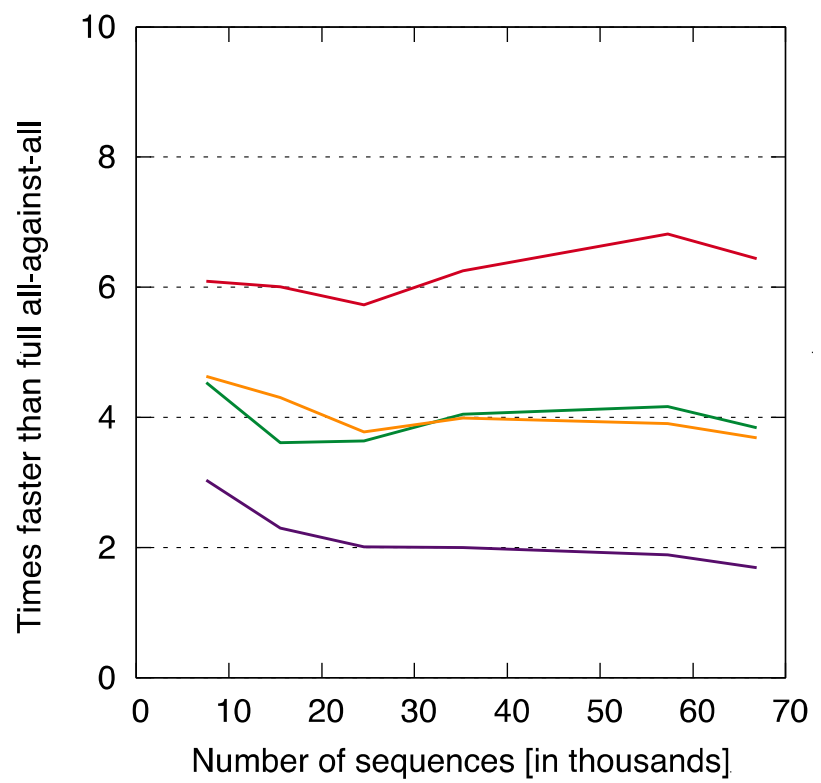

B. Fungi

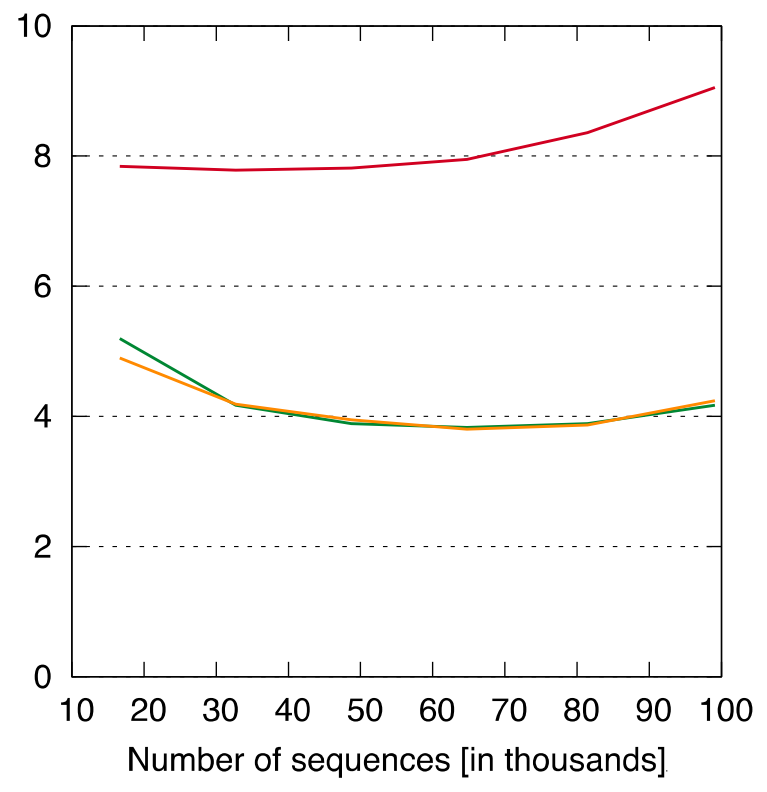

w.o. subsequence homology

1 representative 3 representatives

w/ subsequence homology

Figure 3: Speed up using the new approach. The proposed approach is 2-9x faster, were computed.

252 All four clustering variants decreased runtime compared to the full all-against-all

253 algorithm, with a speedup factor of 2-9 depending on the variant and dataset (Fig 3). 
254 Unsurprisingly, the reduction was strongest in variants considering a single

255 representative sequence per cluster. Likewise, ignoring residue-level homology lead

256 to faster runtime, though the difference was more modest.

257 In terms of performance-which we measured in terms of the proportion of

258 significant pairs from the full all-against-all that were recovered by the new variants

259 (referred to as "recall") or its complement (referred to as "missing pairs")-we

260 observed a clear benefit in taking into account residue-level homology, with recall

261 values consistently $>99.6 \%$ (Fig. 4). The use of three representative sequences per

262 cluster also led to increase in recall, but not nearly to the same extent. The recall

263 values obtained for all variants were consistent across the bacteria and fungi

264 datasets and for different sizes, which suggests that they might hold across other

265 datasets as well. To illustrate the nature of missing homologs, two cases are detailed

266 in the Supplementary materials (Supplementary materials Fig. 3-6).

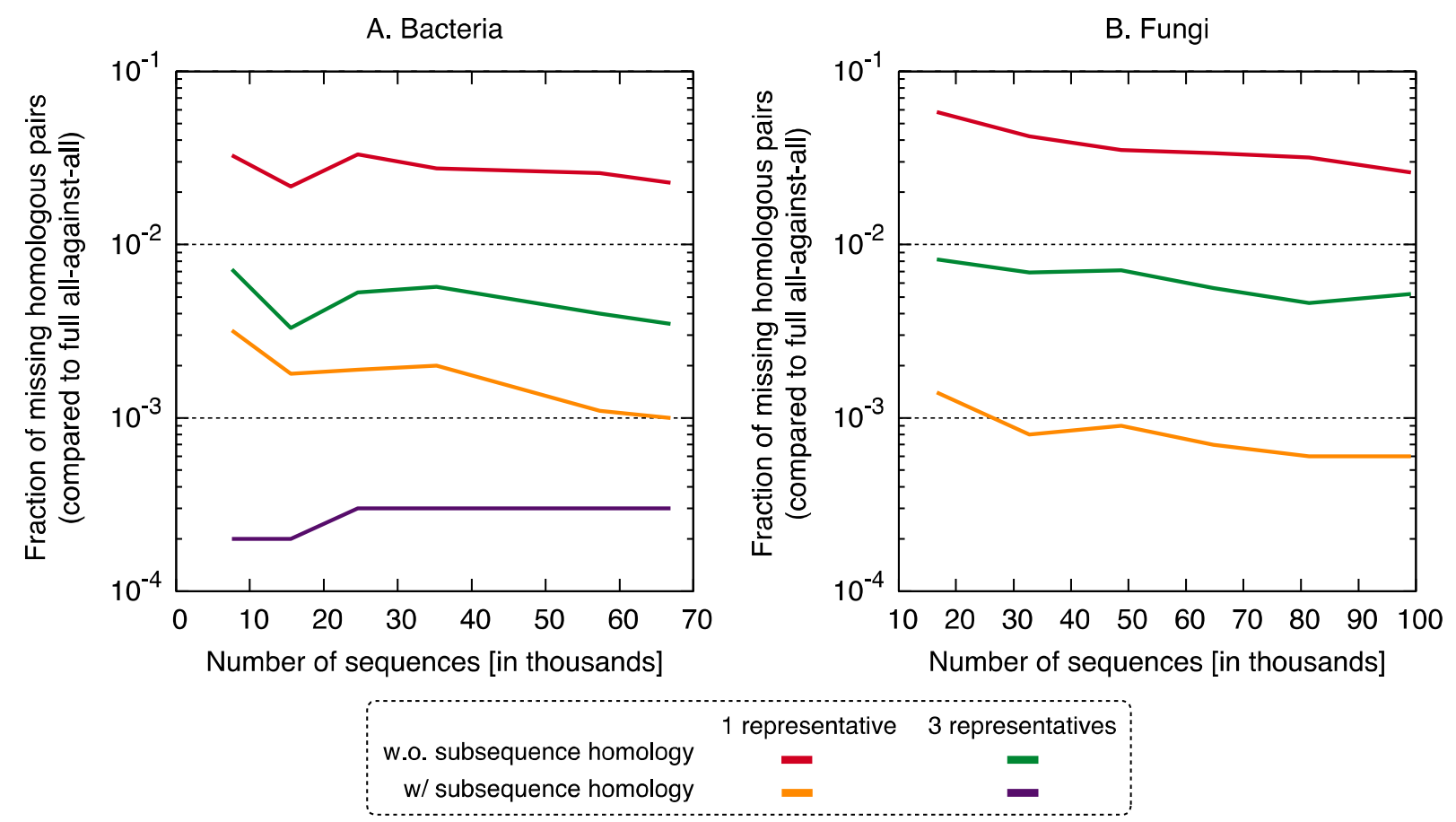

Figure 4: Fraction of the pairs missed when using the proposed algorithm. The new approach misses $0.4-6 \%$ of the pairs from the full all-against-all in its simple variant ( 1 and 3 representatives), and $0.01-0.3 \%$ taking into account sub-sequence homology.

270 Putting runtime and accuracy together, the best results were obtained by taking into

271 account subsequence-level homology using a single representative sequence, which

272 achieved a $\sim 4 x$ speedup while maintaining $>99.6 \%$ recall in both datasets. 
273 To gain insight into the nature of missing pairs, we analysed the distribution of

274 alignment scores of missing pairs compared to that of all significant matches (Fig.

275 5). For all method variants, but especially those taking into account subsequence-

276 level homology, the distribution is heavily skewed toward low-scoring matches. This

277 is favourable, because low-scoring matches are less reliable in the first place and

278 therefore downstream analyses are already designed to cope with missing

279 homologous relationships among distant pairs (e.g. Altenhoff et al. 2013). We also

280 note that the higher recall observed with three representatives instead of one is

281 mainly concentrated on low scoring matches. Furthermore, a per-gene analysis of

282 the rate of missing pairs suggests that errors are distributed quite evenly across all

283 family sizes (Supplementary materials Fig. 7).

A. Bacteria

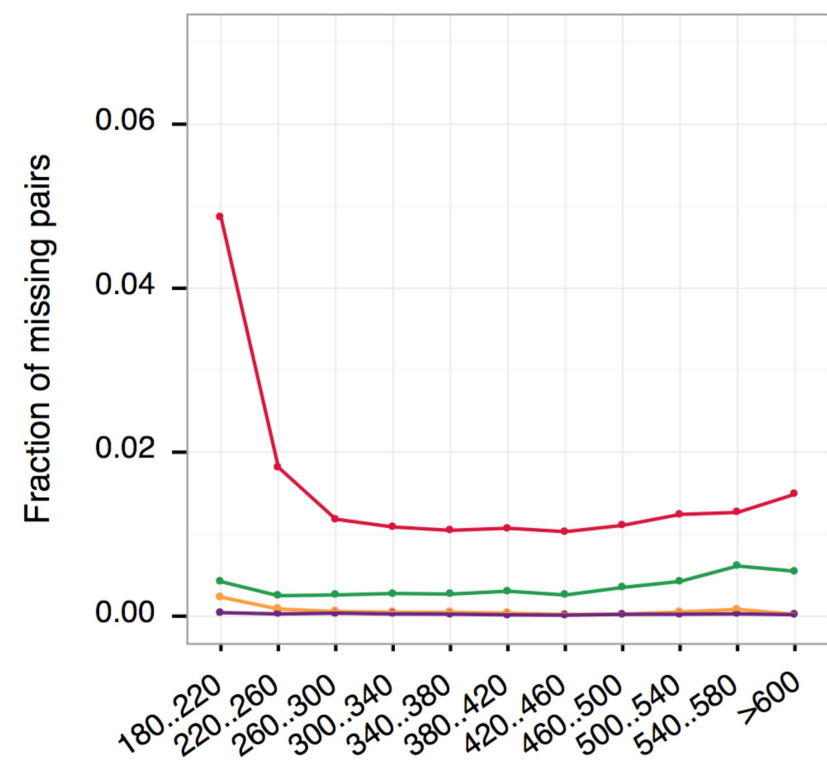

Alignment score [GCB 224 Matrix]
B. Fungi

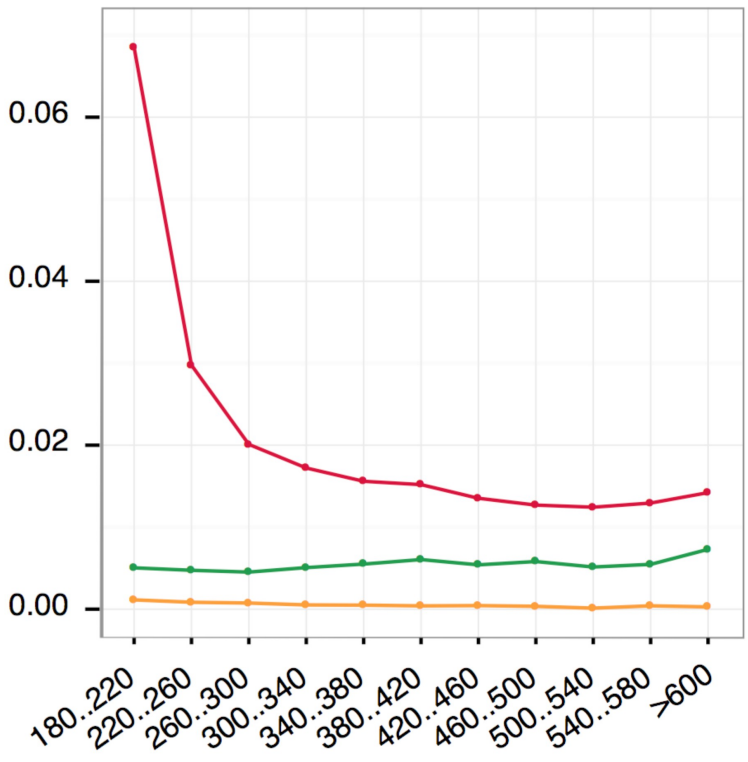

Alignment score [GCB 224 Matrix]

w.o. subsequence homology

1 representative 3 representatives

$\mathrm{w} /$ subsequence homology

Figure 5: Distribution of alignments scores of missing pairs compared with all pairs

("background"). With a single representative, the distribution is heavily skewed toward low-scoring pairs.

287 For any given taxonomic range, adding new genomes and the corresponding new 288 sequences can only increase the number of clusters. However, as the number of 
289 genomes grows, we can expect that an increasing proportion of the sequences will

290 fall into one of the existing clusters. Thus, we should see a tapering in the number of

291 clusters as a function of the number of sequences, which would be favourable in

292 terms of runtime of the algorithm. In our datasets, we could not observe such

293 tapering, and instead the growth in cluster numbers was broadly linear

294 (Supplementary materials Fig. 8-9). This suggests that 12-14 genomes are too few to 295 discern the additional asymptotic benefits of our new approach. A similar conclusion 296 can be drawn from the distribution of cluster size, which is heavily skewed toward 297 very small clusters (Supplementary materials Fig. 10). The large overlap among 298 numerous clusters and the existence of many sequences included in multiple 299 clusters (Supplementary materials Fig. 11) suggests improvement potential by 300 merging some of the clusters (see also Discussion below).

301 To assess the impact of the new clustering approach on orthology inference, we ran 302 the OMA standalone software (Altenhoff et al. 2013) using our best new variant (1 303 representative, with subsequence homology) compared to the full all-against-all as a 304 reference. On the largest Bacteria and Fungi dataset, the proportion of orthologs 305 that were recovered was $99.71 \%$ and $99.87 \%$ respectively. This is slightly lower than 306 the proportion of recovered homologs (99.9\% and $99.94 \%)$, but remains very high.

307 Furthermore, to test whether large genomes and/or multidomain proteins could 308 affect the performance of the new approach, we conducted an additional 309 comparison on a genome set containing two vertebrates (Human and Xenopus 310 tropicalis), one plant (Arabidopsis thaliana) and three unicellular organisms 311 (Plasmodium falciparum, Saccharomyces cerevisiae, and Bacillus subtilis). We 312 applied the variant with one representative and subsequence homology. It resulted 313 in a considerably higher speedup factor of 12.05 , with a recall of $99.94 \%$. This 314 suggests that the new approach behaves well in the presence of large genomes and 315 numerous multi-domain proteins as well.

316 Finally, we sought to compare the new approach to the state-of-the-art $k$-mer 317 clustering approaches UCLUST (Edgar 2010) and kClust (Hauser et al. 2013). As 318 elaborated in the introduction, these methods have been developed to cluster 319 closely related sequences, but given their similar conceptual ideas (clustering, use 320 of representative sequences), they provide an interesting point of comparison. As 
321 expected, these approaches were orders of magnitude faster (speedup 500-1900x

322 for kClust, over $6000 x$ for UCLUST; Fig. 6 ), but their recall was typically in the single

323 percentage points (6.62-13.52\% for kClust, 3.16-10.37\% for UCLUST), even with

324 inclusive parameters (Fig. 7; Supplementary File 2).

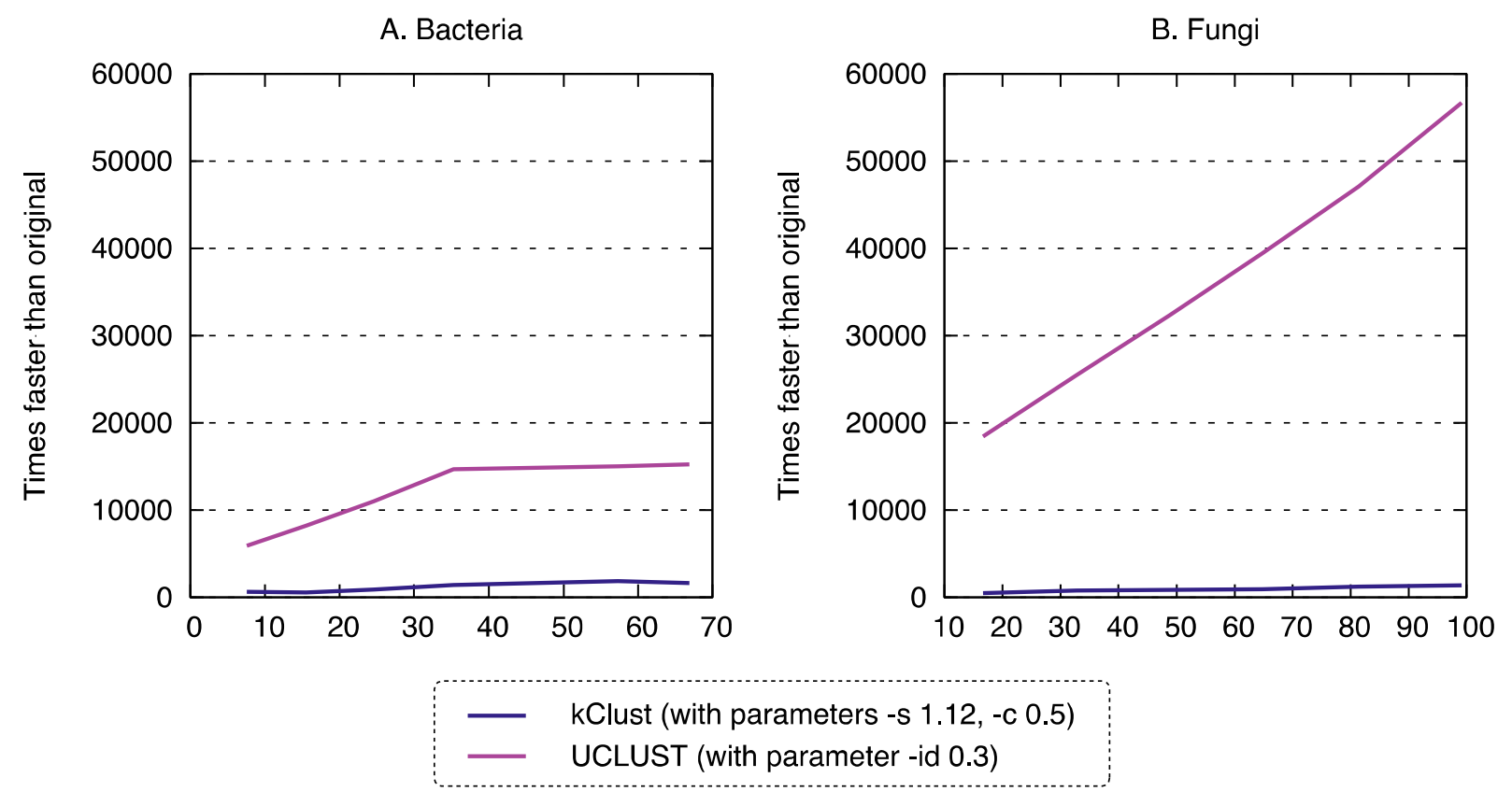

325 Figure 6: Runtime of typical $\boldsymbol{K}$-mer approaches. kClust and UCLUST are several orders of 326 magnitude faster than full all-against-all.
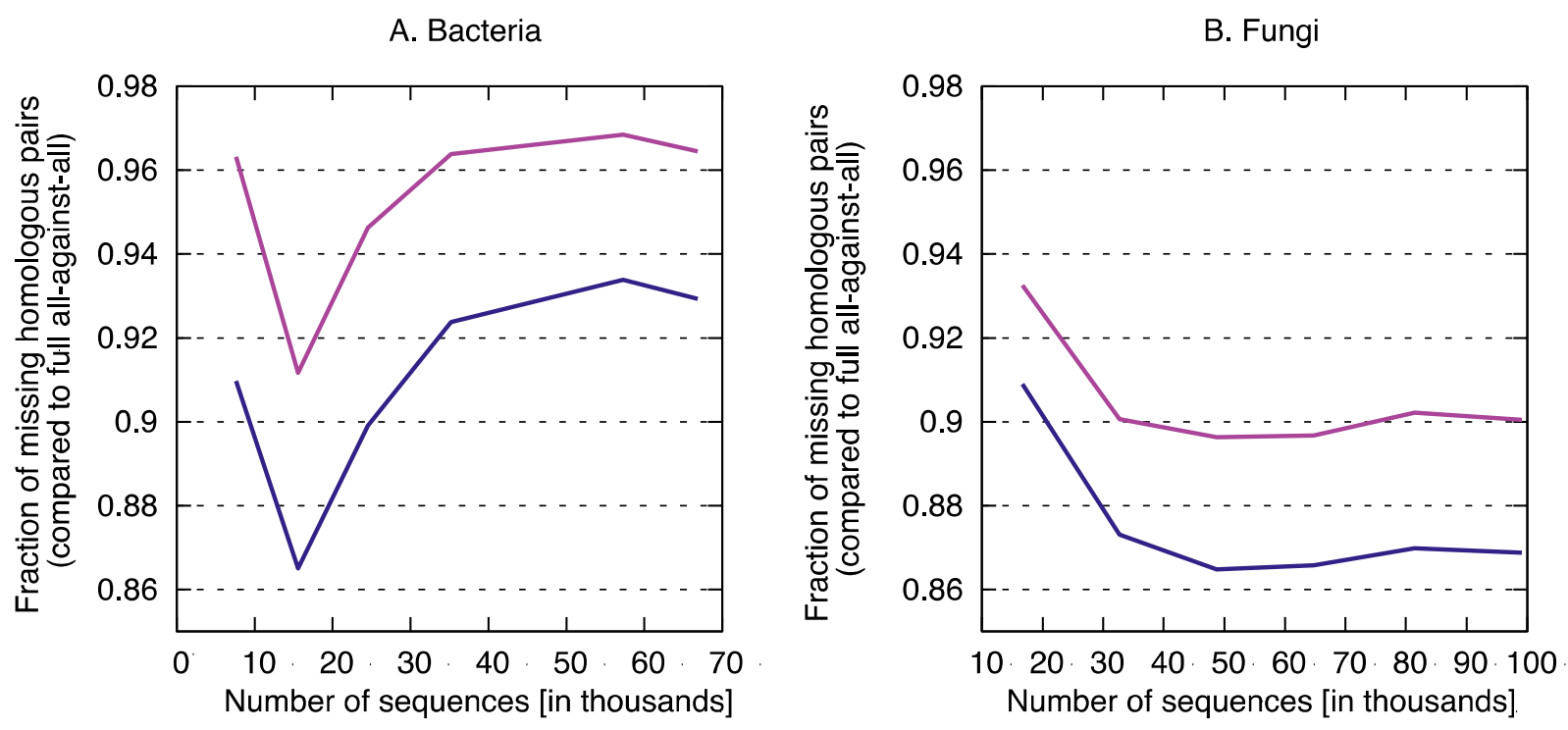

— $\quad$ kClust (with parameters -s 1.12, -c 0.5)

UCLUST (with parameter -id 0.3) 
Fig. 7. Recall of typical K-mer approaches. kClust and UCLUST only recover 3-14\% of the sensitive parameter settings (see Materials and Methods for details).

\section{Discussion and outlook}

331 The all-against-all phase is at the basis of many orthology inference algorithms but 332 due to its quadratic time complexity in terms of number of sequences, the all333 against-all phase can be a bottleneck. This work suggests that the transitivity of 334 homology can be exploited to substantially speedup homology inference while 335 maintaining sensitivity-provided homology is defined in terms of consistent 336 subsequence stretches.

337 The datasets used in this study are challenging, with median distance of 146 PAM 338 (1.46 substitution per site on average) in the bacteria dataset and 149 PAM in the 339 fungi dataset (Supplementary materials Fig. 2). This implies that half of homologous pairs have less than $30 \%$ sequence identity. At such high levels of divergence, $k$-mer based methods perform poorly compared to all-against-all dynamic programming 342 alignment. In contrast, our transitivity clustering approach performed well. It is likely 343 to perform even better on evolutionarily closer sets of taxa, such as vertebrate 344 species or flowering plant species.

345 The speedup we observed with the new approach is substantial. But because the 346 number of clusters increased roughly linearly with the number of genomes in our 347 datasets of up to 14 genomes, the overall time complexity still grows quadratically 348 in this range. However, as rarefaction curves show (e.g. Mira et al. 2010), the growth 349 in the number of homologous clusters typically tapers off. Thus, it is possible that 350 the asymptotic complexity of the new procedure is subquadratic in the number of 351 species. This will need to be confirmed in future work.

352 Apart from the full dynamic programming alignment comparison with cluster 353 representatives and the subsequence-level homology consideration, our approach

354 distinguishes itself from most clustering methods in that it allows sequences to 355 match to several clusters. If homology was always uniform across the whole 356 sequence length and could be perfectly inferred from present-day sequences, each 357 sequence would belong to one and only one homologous cluster. In practice 
358 however, these assumptions do not hold: gene fusion can result in sequences that

359 have homologous relationships across multiple clusters; some homologs have

360 diverged too far to be inferred as such, thus resulting in cluster fragmentation. By

361 allowing for sequences to belong to multiple clusters, our method is robust to these 362 complications.

363 Though the present study focuses on Smith-Waterman dynamic programming 364 alignments, a similar clustering approach would be possible with the faster but less 365 sensitive BLAST (Altschul et al. 1997). One complication with BLAST is however that 366 the sequence database needs to be re-indexed whenever a sequence is added. To 367 mitigate this, one could add new representative sequences in batches, with the 368 additional complication that sequences within each batch would also need to be 369 aligned to one another.

370 A further advantage of the clustering approach is that it also works well in the 371 context of "semi-curated" databases, such as in COGs (Tatusov et al. 2003), Panther 372 (Mi et al. 2013), or PFam (Finn et al. 2014). Indeed, it is conceptually straightforward 373 to let curators optimise particular homologous clusters by fine-tuning representative 374 sequences, coverage and score thresholds on a cluster-by-cluster basis.

375 These promising results notwithstanding, the new approach still has much potential 376 for improvement. To further improve the recall, several ideas could be explored.

377 First, the procedure could merge clusters that have a high proportion of members in 378 common. This could recover some of the missed homologous pairs. Second, the 379 choice of cluster representatives could be improved. Indeed, the current strategy of 380 selecting the first sequence (or the first three sequences) added to the cluster is 381 likely to be suboptimal in most instances. Instead, a better choice of representative 382 would be to try to select a sequence with minimal average distance to all cluster 383 members (a "centroid" sequence). Another idea, suggested by Dr. Ikuo Uchiyama in 384 his peer-review, would be to vary the number of representatives depending on the 385 particular needs of each cluster, e.g. by adding a new member as a representative if 386 its similarity score with the existing representative(s) is below a certain threshold. 387 Third, as alternative to representative sequences, clusters could be represented by 388 Hidden Markov model profiles. Such approach, at the basis of well-established 389 methods such as PSI-Blast (Altschul et al., 1997), HMMER (Eddy, 2011), or HHBlitz 
390 (Remmert et al., 2011), is likely be more sensitive than pairwise alignment with a

391 single representative sequence. The profiles could be periodically updated as new

392 sequences are assigned to the cluster.

393 We also see potential to further improve the speed of the new approach. First, 394 merging homologous clusters can lead to a speed improvement because the cost of 395 assigning sequences to clusters grows linearly in the number of clusters. This needs 396 to be done carefully, because excessive merging - the merging of clusters containing 397 a substantial number of non-homologous pairs-can reduce the efficiency of the 398 within-cluster all-against-all, whose time complexity grows quadratically in the 399 number of sequences. Second, it may be possible to optimise the assignment of 400 sequences to clusters by identifying clusters that are so different to one another that 401 they are practically mutually exclusive and thus inclusion into one implies exclusion 402 from the other. An empirical way of establishing such mutual exclusivity would be to 403 keep track of the number or proportion of sequences belonging to both clusters.

404 Finally, the new approach could be parallelised. One way of parallelising the 405 assignment of sequences to clusters would be to use a Publisher-Subscriber model 406 (Eugster et al. 2003): a "master" process would start the analysis of a new sequence 407 by distributing it to a set of "workers", each responsible to compare the sequence to 408 a subset of all existing clusters. Each worker would thus align the new sequence to 409 its designated subset of clusters and reports back significant matches and their 410 associate sequence ranges (subsequence-level coverage). Once the master process

411 has received this information from all workers, it would ensure that the new

412 sequence is fully covered by matches to existing clusters, and else it would generate 413 a new cluster with that sequence as representative. As for the within-cluster all414 against-all comparisons, they could be straightforwardly parallelised thanks to the 415 lack of dependency among all pairs.

416 Meanwhile, a serial implementation of our best variant (accounting for subsequence417 level homology using a single representative sequence) is available as part of the 418 open source OMA standalone package (http://omabrowser.org/standalone).

\section{$419 \quad$ Acknowledgements}


420 This article is dedicated to Gaston H. Gonnet on the occasion of his retirement. We

421 thank Kevin Gori, Henning Redestig, Nives Škunca, Bartlomiej Tomiczek for useful

422 feedback on the manuscript, as well as Odile Lecompte, Ikuo Uchiyama and one

423 anonymous reviewers for useful feedback on the manuscript.

\section{Supplementary Materials}

4250 PDF containing supplementary figures 1-11 (appended to this preprint for

426 convenience)

427 Supplementary File 1: list of species, including taxonomic ID and genome source and

428 version.

429 Supplementary File 2: detailed comparisons with kClust and UCLUST.

430

431

432

433

434

435

436

437

438

439

440

441

442

443

444

445

446

447

448

449

450

451

452

453

454

455

456

457

458

459

460

\section{Bibliography}

Altenhoff A.M., Dessimoz C. 2012. Inferring Orthology and Paralogy. In: Anisimova M., editor. Evolutionary Genomics. Humana Press. p. 259-279.

Altenhoff A.M., Gil M., Gonnet G.H., Dessimoz C. 2013. Inferring hierarchical orthologous groups from orthologous gene pairs. PLoS One. 8:e53786.

Altenhoff A.M., Schneider A., Gonnet G.H., Dessimoz C. 2011. OMA 2011: orthology inference among 1000 complete genomes. Nucleic Acids Res. 39:D289-94.

Altenhoff A.M., Studer R. a., Robinson-Rechavi M., Dessimoz C. 2012. Resolving the ortholog conjecture: orthologs tend to be weakly, but significantly, more similar in function than paralogs. PLoS Comput. Biol. 8:e1002514.

Altschul S.F., Madden T.L., Schäffer A.A., Zhang J., Zhang Z., Miller W., Lipman D.J. 1997. Gapped BLAST and PSI-BLAST: a new generation of protein database search programs. Nucleic Acids Res. 25:3389-3402.

DeLuca T.F., Cui J., Jung J.-Y., St Gabriel K.C., Wall D.P. 2012. Roundup 2.0: enabling comparative genomics for over 1800 genomes. Bioinformatics. 28:715-716.

Dessimoz C., Cannarozzi G., Gil M., Margadant D., Roth A., Schneider A., Gonnet G. 2005. OMA, A Comprehensive, Automated Project for the Identification of Orthologs from Complete Genome Data: Introduction and First Achievements. RECOMB 2005 Workshop on Comparative Genomics.61-72.

Dessimoz C., Gabaldón T., Roos D.S., Sonnhammer E.L.L., Herrero J., Quest for Orthologs Consortium. 2012. Toward community standards in the quest for orthologs. Bioinformatics. 28:900-904.

Edgar R.C. 2010. Search and clustering orders of magnitude faster than BLAST. Bioinformatics. 26:2460-2461.

Eugster P.T., Felber P.A., Guerraoui R., Kermarrec A.-M. 2003. The Many Faces of Publish/Subscribe. ACM Comput. Surv. 35:114-131.

Finn R.D., Bateman A., Clements J., Coggill P., Eberhardt R.Y., Eddy S.R., Heger A., Hetherington K., Holm L., Mistry J., Sonnhammer E.L.L., Tate J., Punta M. 2014. Pfam: the protein families database. Nucleic Acids Res. 42:D222-30.

Fitch W.M. 1970. Distinguishing homologous from analogous proteins. Syst. Zool. 19:99-113. 
461

462

463

464

465

466

467

468

469

470

471

472

473

474

475

476

477

478

479

480

481

482

483

484

485

486

487

488

489

490

491

492

493

494

495

496

497

498

499

500

501

502

503

504

505

506

507

508

509

510

511

512

513

Gabaldón T., Koonin E.V. 2013. Functional and evolutionary implications of gene orthology. Nat. Rev. Genet. 14:360-366.

Gaudet P., Livstone M.S., Lewis S.E., Thomas P.D. 2011. Phylogenetic-based propagation of functional annotations within the Gene Ontology consortium. Access. 12.

Gonnet G.H., a Cohen M., a Benner S. 1992. Exhaustive matching of the entire protein sequence database. Science. 256:1443-1445.

Hauser M., Mayer C.E., Söding J. 2013. kClust: fast and sensitive clustering of large protein sequence databases. BMC Bioinformatics. 14:248.

Li W., Godzik A. 2006. Cd-hit: a fast program for clustering and comparing large sets of protein or nucleotide sequences. Bioinformatics. 22:1658-1659.

Lynch M. 2000. The Evolutionary Fate and Consequences of Duplicate Genes. Science. 290:1151-1155.

Metzker M.L. 2009. Sequencing technologies - the next generation. Nat. Rev. Genet. 11:31-46.

Mi H., Muruganujan A., Thomas P.D. 2013. PANTHER in 2013: modeling the evolution of gene function, and other gene attributes, in the context of phylogenetic trees. Nucleic Acids Res. 41:D377-86.

Mira A., Martín-Cuadrado A.B., D'Auria G., Rodríguez-Valera F. 2010. The bacterial pan-genome: a new paradigm in microbiology. International Microbiology. 13:45-57.

Pagani I., Liolios K., Jansson J., Chen I. 2012. The Genomes OnLine Database (GOLD) v. 4: status of genomic and metagenomic projects and their associated metadata. Nucleic acids.

Patterson C. 1988. Homology in classical and molecular biology. Mol. Biol. Evol. 5:603-625.

Powell S., Forslund K., Szklarczyk D., Trachana K., Roth A., Huerta-Cepas J., Gabaldón T., Rattei T., Creevey C., Kuhn M., Jensen L.J., von Mering C., Bork P. 2013. eggNOG v4.0: nested orthology inference across 3686 organisms. Nucleic Acids Res. 42:D231-9.

Rentzsch R., Orengo C. a. 2009. Protein function prediction--the power of multiplicity. Trends Biotechnol. 27:210-219.

Roth A.C.J., Gonnet G.H., Dessimoz C. 2008. Algorithm of OMA for large-scale orthology inference. BMC Bioinformatics. 9:518.

Schreiber F., Sonnhammer E.L.L. 2013. Hieranoid: hierarchical orthology inference. J. Mol. Biol. 425:2072-2081.

Smith T.F., Waterman M.S. 1981. Identification of common molecular subsequences. J. Mol. Biol. 147:195-197.

Sonnhammer E.L.L., Gabaldón T., Sousa da Silva A.W., Martin M., Robinson-Rechavi M., Boeckmann B., Thomas P.D., Dessimoz C., the Quest for Orthologs consortium. 2014. Big data and other challenges in the quest for orthologs. Bioinformatics. Advanced access online.

Szalkowski A., Ledergerber C., Krähenbühl P., Dessimoz C. 2008. SWPS3 - fast multithreaded vectorized Smith-Waterman for IBM Cell/B.E. and x86/SSE2. BMC Res. Notes. 1:107.

Tatusov R.L., Fedorova N.D., Jackson J.D., Jacobs A.R., Kiryutin B., Koonin E.V., Krylov D.M., Mazumder R., Mekhedov S.L., Nikolskaya A.N., Sridhar Rao B., Smirnov S., Sverdlov A.V., Vasudevan S., Wolf Y.I., Yin J.J., Natale D. a. 2003. The COG database: an updated version includes eukaryotes. BMC Bioinformatics. 4:41.

Tatusov R.L. 1997. A Genomic Perspective on Protein Families. Science. 278:631637.

Vilella A.J., Severin J., Ureta-Vidal A., Heng L., Durbin R., Birney E. 2008. EnsemblCompara GeneTrees: Complete, duplication-aware phylogenetic trees 

in vertebrates. Genome Res. 19:327-335.

515 Waterhouse R.M., Tegenfeldt F., Li J., Zdobnov E.M., Kriventseva E.V. 2013. OrthoDB:

516

517 a hierarchical catalog of animal, fungal and bacterial orthologs. Nucleic Acids Res. 41:D358-65. 


\section{Figure 1}

Fig 1: Diagram of potential problems with transitivity of homology.

(A) The transitivity of homology does not hold if the residues involved are inconsistent. (B) Homologous relationships can be missed if significant sequence parts are not covered by the representative sequence. To address this problem, our approach would create a new cluster, with the sequence that is not fully covered as its representative.

A

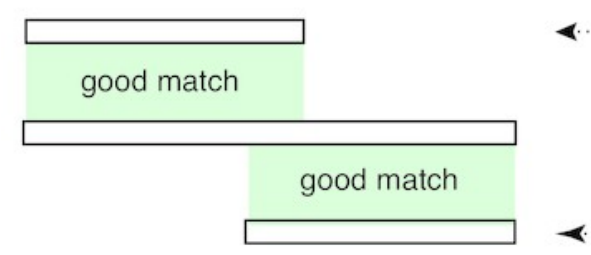

Transitivity of homology does not hold across inconsistent subsequences
B

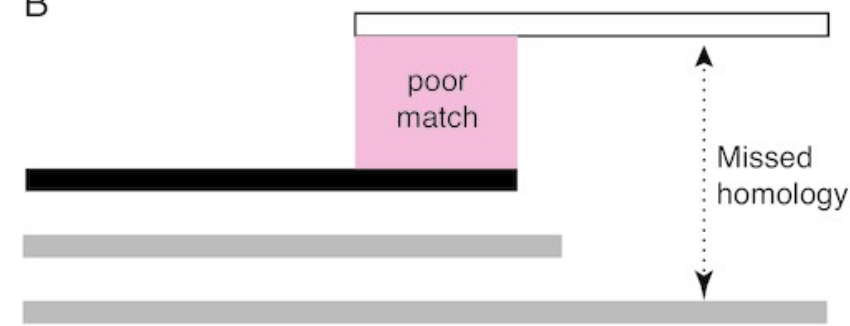

Non-representative cluster member 


\section{Figure 2}

Fig. 2: Comparison between the current and the new approach.

Diagram of (a) the current OMA all-against-all step and (b) the new approach with homologous clustering and sequence coverage. In the proposed approach, the sequences are first clustered and then the all-against-all is run only within clusters. The overall number of computations is considerably reduced, but some homologous relationships can be missed.

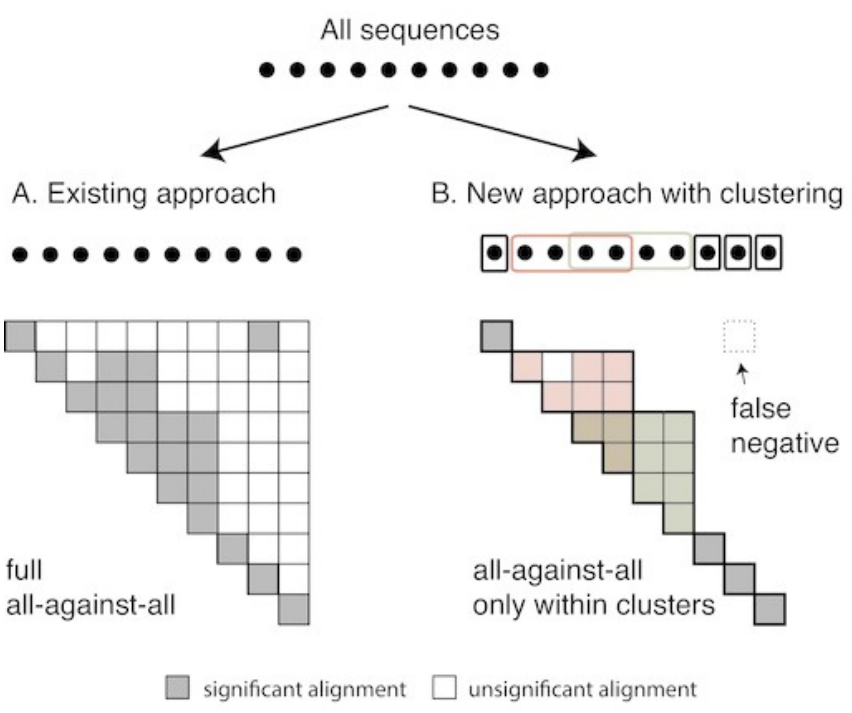




\section{Figure 3}

Fig. 3: Speed up using the new approach.

The proposed approach is 2-9x faster, depending on datasets and variants. On the fungi dataset, only the three fastest variants were computed.
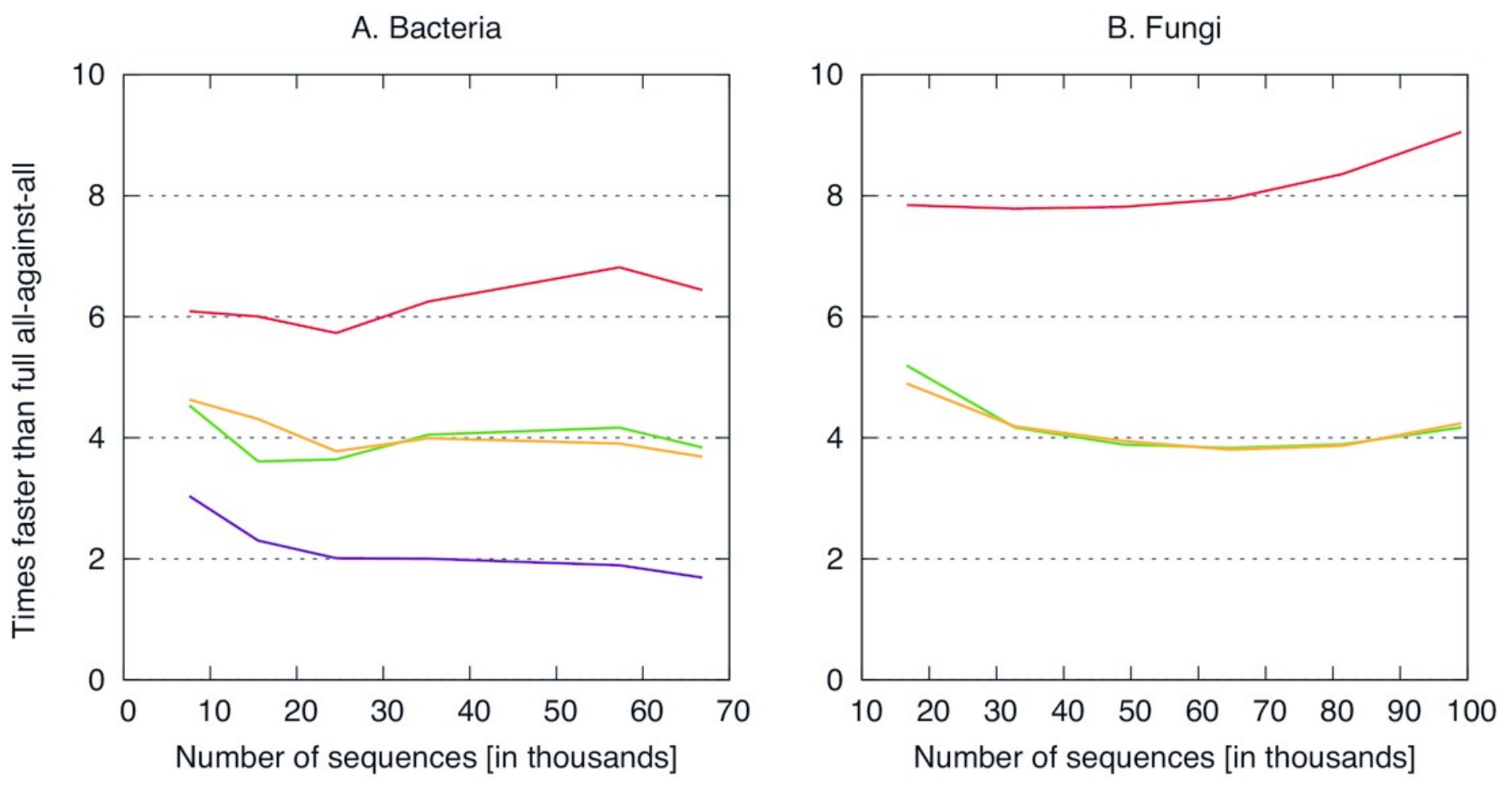

w.o. subsequence homology

1 representative 3 representatives

$\mathrm{w} /$ subsequence homology 


\section{Figure 4}

Figure 4: Fraction of the pairs missed when using the proposed algorithm.

The new approach misses $0.4-6 \%$ of the pairs from the full all-against-all in its simple variant (1 and 3 representatives), and $0.01-0.3 \%$ taking into account sub-sequence homology.
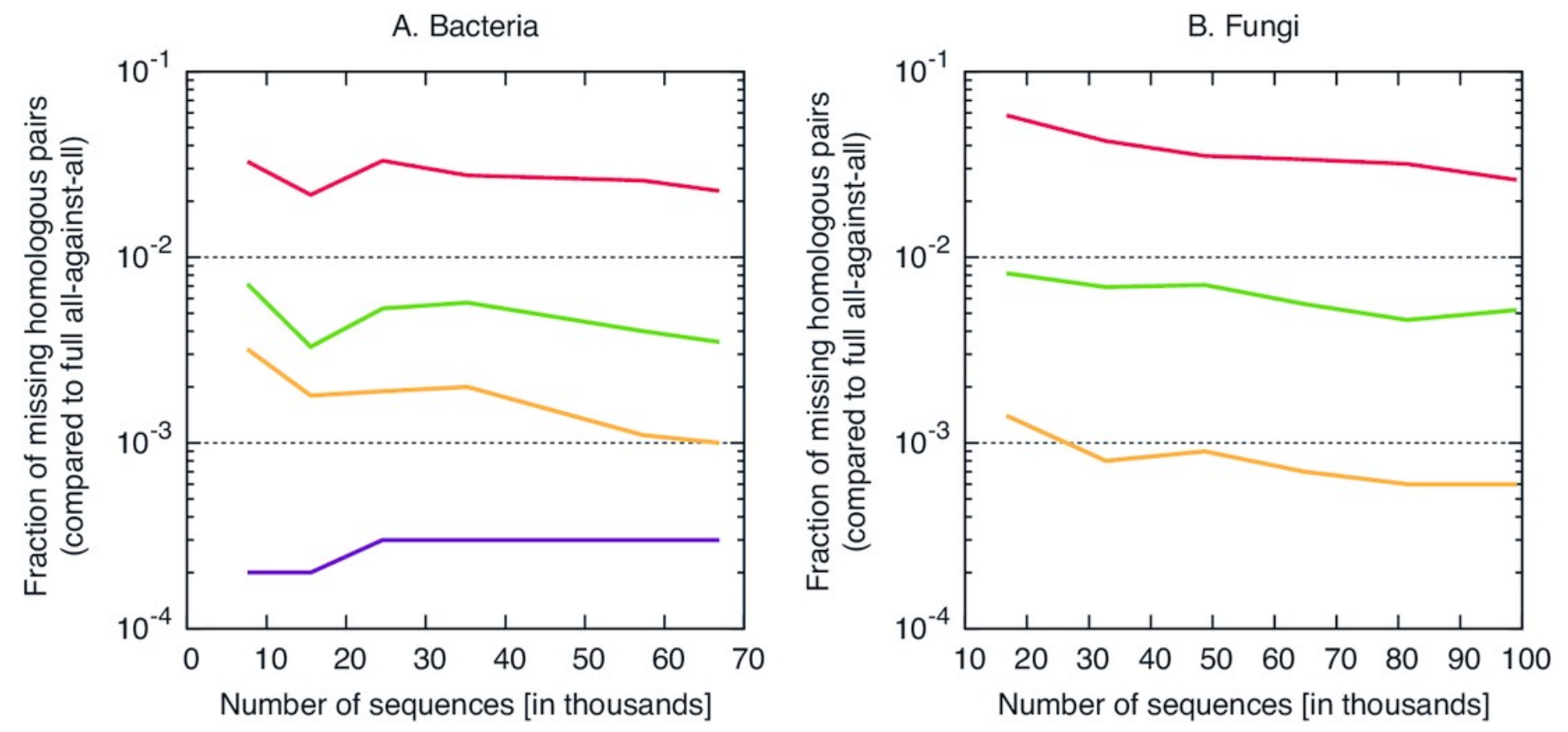

w.o. subsequence homology 1 representative 3 representatives
w/ subsequence homology




\section{Figure 5}

Figure 5: Distribution of alignments scores of missing pairs compared with all pairs ("background").

With a single representative, the distribution is heavily skewed toward low-scoring pairs.

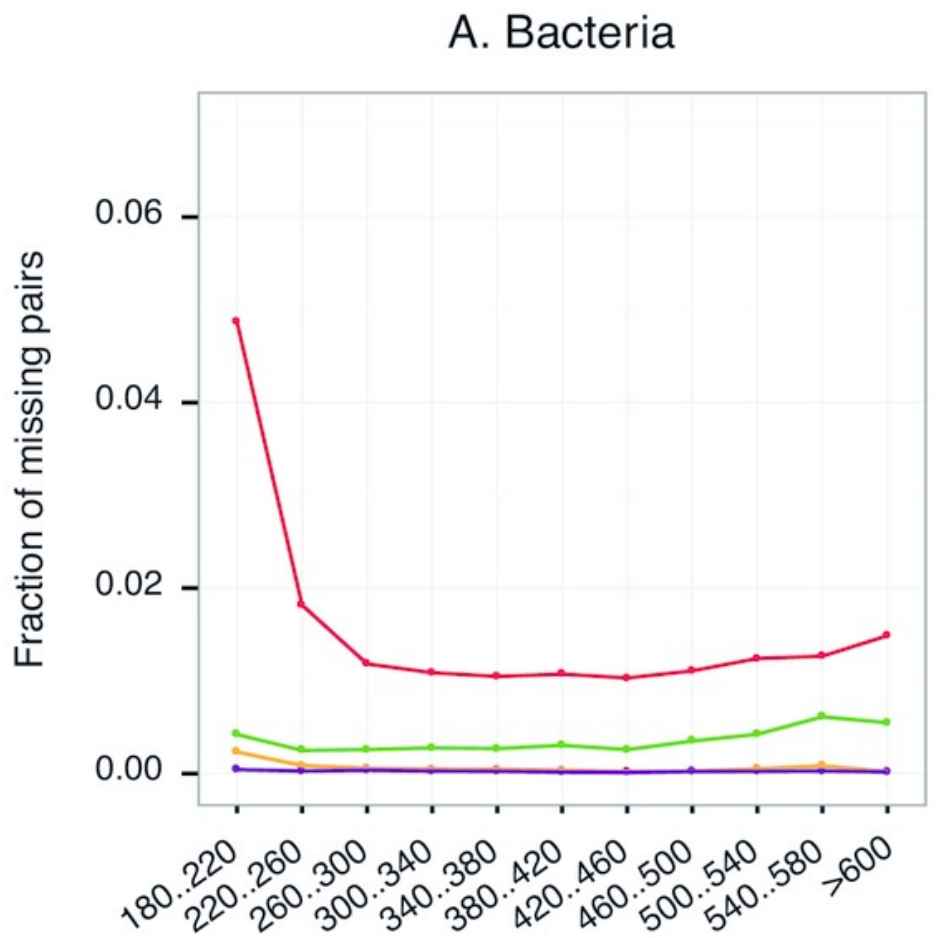

Alignment score [GCB 224 Matrix]

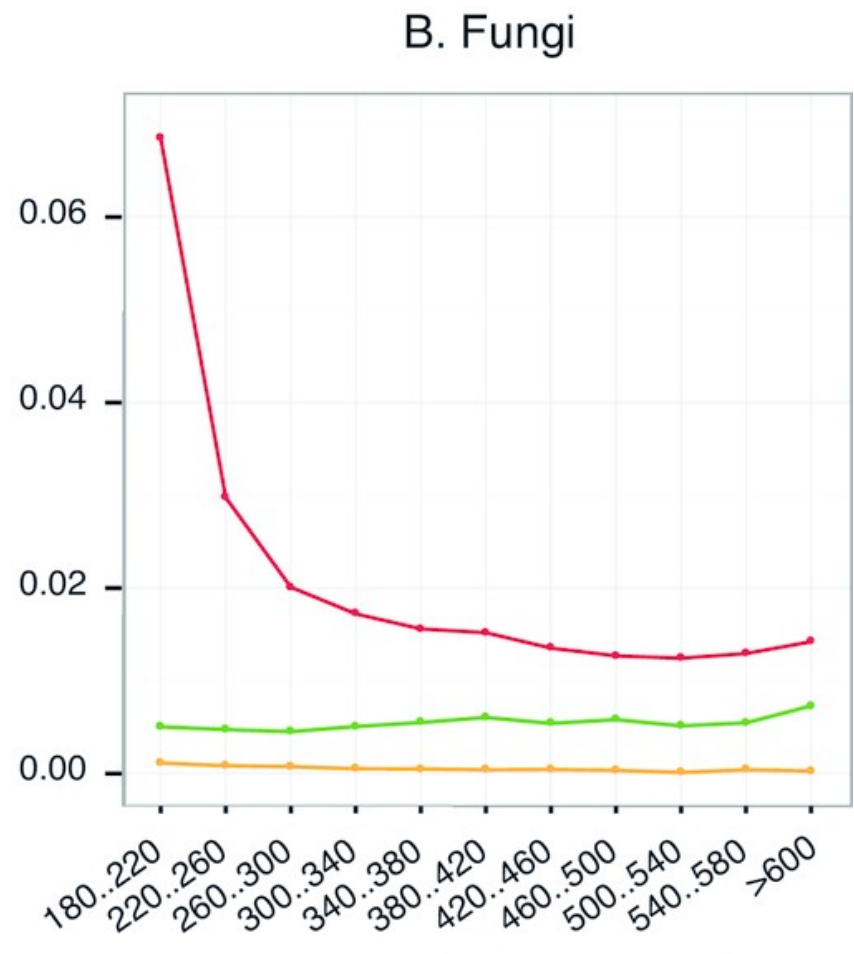

Alignment score [GCB 224 Matrix]

w.o. subsequence homology

1 representative 3 representatives

w/ subsequence homology 


\section{Figure 6}

Figure 6: Runtime of typical K-mer approaches.

kClust and UCLUST are several orders of magnitude faster than full all-against-all.

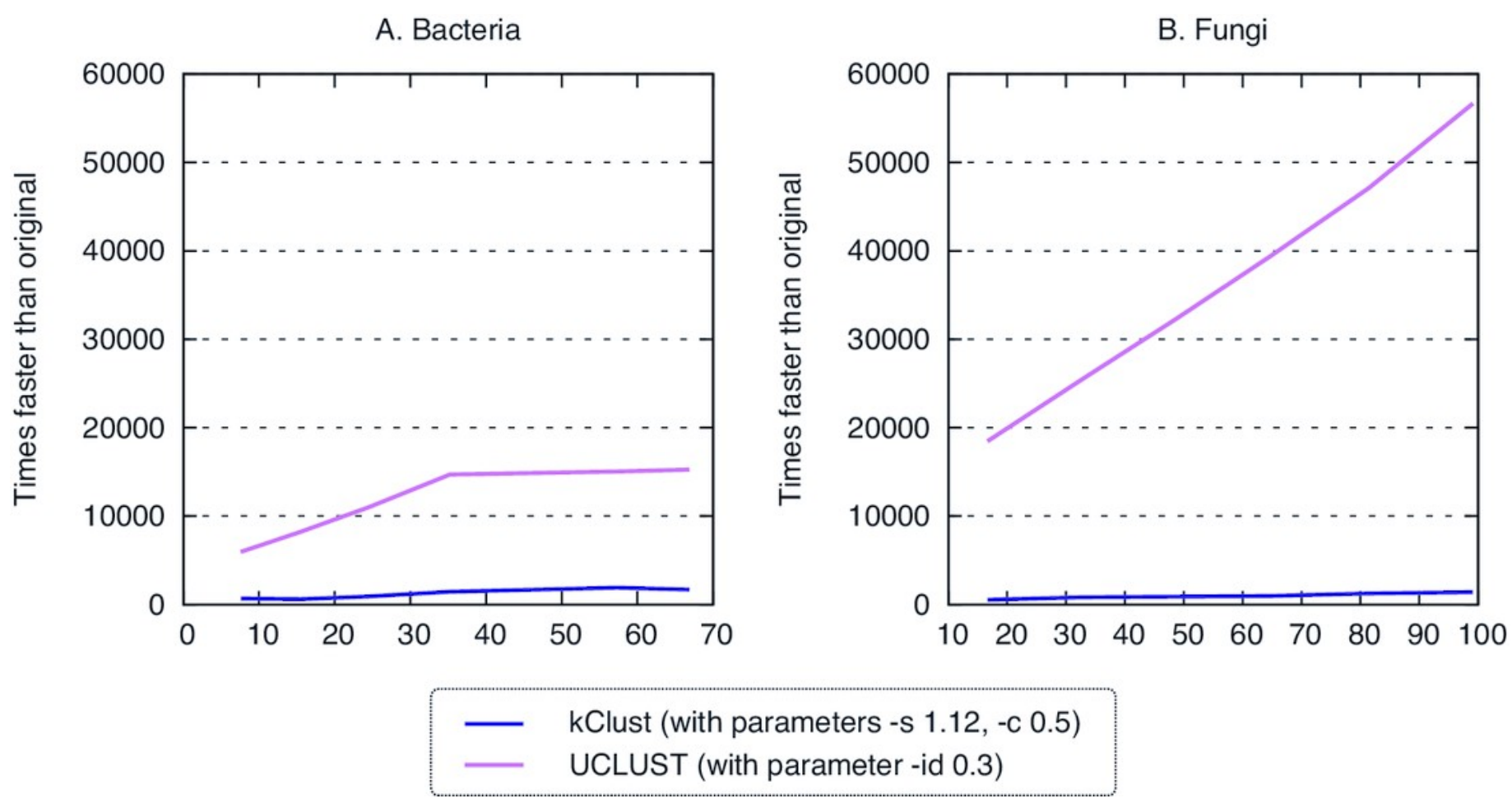




\section{Figure 7}

Fig. 7: Recall of typical K-mer approaches.

kClust and UCLUST only recover $3-14 \%$ of the homologous pairs identified by the full allagainst-all procedure, even when they are run with sensitive parameter settings (see Materials and Methods for details).

A. Bacteria

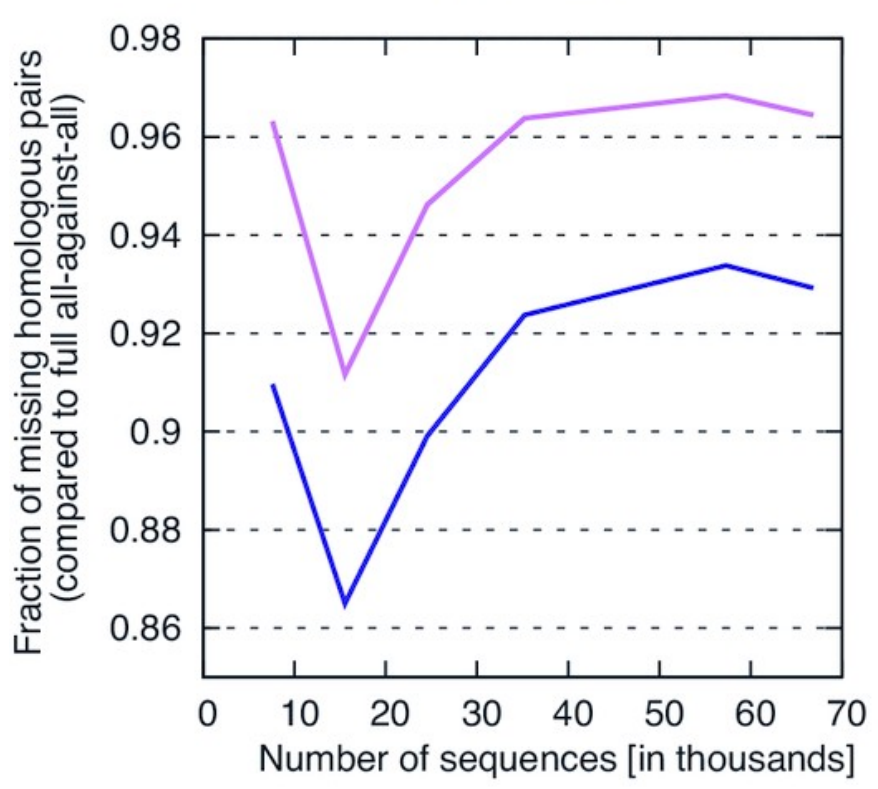

B. Fungi

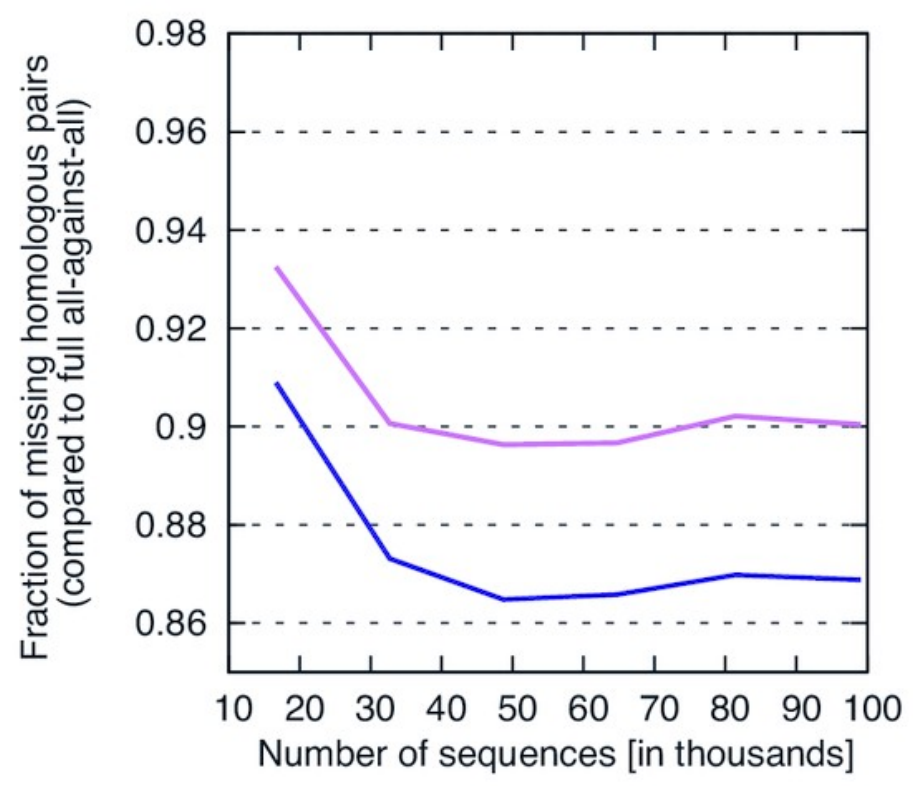

— $\quad$ kClust (with parameters -s 1.12, -c 0.5)

_ UCLUST (with parameter -id 0.3) 